\title{
Evaluación y diagnóstico de la disfonía en niños
}

\section{Assesment and diagnosis of dysphonia in children}

\author{
Dr. Christian Olavarría Leiva \\ Otorrinolaringólogo \\ Hospital Clínico Universidad de Chile \\ Clínica Santa María
}

\section{Dr. Pedro Cortez Villagrán}

Residente Otorrinolaringología

Servicio Otorrinolaringología

Hospital Clínico Universidad de Chile

\begin{abstract}
ASSESMENT AND DIAGNOSIS OF DYSPHONIA IN CHILDREN
Dysphonia in children is a common symptom but not a cause of common query in pediatrics, and usually is underestimated by parents and physicians. Most of vocal symptoms are caused by vocal abuse, however hoarseness can be a symptom of a relevant health problem and even severe in children. The following paper aims to expand the diagnostic range in this group of patients.
\end{abstract}

Keywords: dysphonia, vocal nodules, children

\section{RESUMEN}

La disfonía en el niño es un síntoma frecuente, no así un motivo de consulta habitual, ya que con frecuencia suele ser subestimado por padres y médicos. Si bien la mayoría de los cuadros son debido a hiperfunción o mal uso de la voz, la disfonía en el niño puede ser síntoma de un problema de salud relevante e incluso grave. La siguiente publicación busca ampliar el abanico diagnóstico en este grupo de pacientes.

Palabras clave: disfonía, nódulos vocales, niño.

\section{INTRODUCCIÓN}

La disfonía se define como un trastorno caracterizado por la alteración de la calidad de la voz, tono, volumen o esfuerzo vocal que limita la comunicación o reduce la calidad de vida relacionada con la voz (1).

Los síntomas vocales están presentes en el 6-23\% de los niños entre los 4 y 12 años de edad y estos se deben a causas inflamatorias, infecciosas, congénitas, traumáticas, neurológicas, iatrogénicas o funcionales $(2,3)$. En la mayoría de los casos, este problema no es percibido con preocupación por los padres por lo tanto no consultan de forma precoz. Tanto los niños como sus padres se "acostumbran" a esta voz alterada del niño, siendo frecuentemente advertida por alguien externo, como el profesor. Es aconsejable una temprana identificación de los trastornos de la voz pediátricos, dado que estos pueden progresar a disfunciones crónicas si no se tratan $(4,5)$.

\section{DIFERENCIAS LARINGE INFANTIL VS ADULTO}

La laringe infantil es más pequeña y se ubica en una posición más alta que en el adulto, con el cricoides a nivel de la vertebra C3-

\section{Correspondencia:}

Dr. Christian Olavarría

Dirección: Avda. Santa María 0410, Providencia

Santiago de Chile

e-mail: christian.olavarria@gmail.com

ISSN 0718-33X. Derechos reservados.
C4. La epiglotis tiene una forma curva (en omega). Las cuerdas vocales (CV) son más cortas y tienen una proporción diferente. En el adulto los 2/3 anteriores del pliegue vocal tienen función fonatoria y el 1/3 posterior tiene función respiratoria. En cambio en el niño esta proporción es 1/1. La estructura histológica de la CV en el niño es inmadura, carente de la estratificación,lo cual podría ser relevante para la cicatrización cordal (6).

\section{ETIOPATOGENIA}

El fonotrauma puede ocurrir debido a factores psicosociales como la hiperactividad o impulsividad, o debido a historia de Ilanto excesivo, siendo esto frecuente en la población pediátrica (7). Durante el juego, los niños suelen aumentar la intensidad de la voz, con mayor esfuerzo y tensión muscular cervical. Estos hábitos son característicos en las disfonías musculoesqueléticas o hipercinéticas y propician la aparición de nódulos vocales (NV), producto del continuo y abrupto golpe de las CV durante la fonación. Los análisis histológicos de los NV han demostrado proliferación de las capas epiteliales, engrosamiento de la membrana basal y abundante fibronectina en la lámina propia (7). Además de NV existen otras lesiones cordales, que pueden diagnosticarse en niños disfónicos, tales como quistes, parálisis de CV y papilomatosis. También es posible encontrar pequeñas malformaciones congénitas en los pliegues vocales (7-9).

\section{FACTORES DE RIESGO}

Dentro de los factores de riesgo para el desarrollo de disfonía en niños se incluyen hipoacusia, infecciones del tracto respiratorio 
alto, familias numerosas y habitar en un hogar ruidoso. Además, la excesiva demanda vocal, la competencia por ganar el espacio para hablar, la tos persistente y el carraspeo, son todos posibles fenómenos que explicarían la prevalencia de disfonía infantil (6).

\section{ETIOLOGÍA}

\section{Funcional}

Se produce en el contexto de indemnidad anatómica de la laringe. Generalmente asociado a mal uso/abuso del sistema vocal, en que pueden identificarse factores psicológicos involucrados y que pueden desencadenar lesiones benignas si no se maneja a tiempo la condición (5).

\section{Orgánica de base funcional}

Son lesiones anatómicas benignas de la laringe, desencadenadas por abuso/mal uso vocal, destacando los NV, pólipos, quistes submucosos, edema de Reinke y úlcera/granuloma cordal.

Los NV (Figura 1) son la causa más común de disfonía en niños, siendo más frecuentes en varones (2:1) y la severidad de la disfonía tiene relación con el tamaño del nódulo. En general son de manejo foniátrico y la cirugía rara vez está indicada, a pesar de que esto no está avalado por la literatura según una revisión Cochrane reciente, dado la inexistencia de estudios randomizados. En aquel grupo refractario a terapia e higiene vocal, podría considerarse la realización de una laringoscopía directa. Bouchayer et al describen la presencia de quistes, sulcus y pólipos en niños con diagnóstico previo de nódulos, e incluso microwebs (microsinequia) en comisura anterior (1013).

\section{Figura $\mathbf{N}^{0} 1$}

Nódulos vocales: disgenesia vascular en tercio medio de cuerdas vocales izquierda.

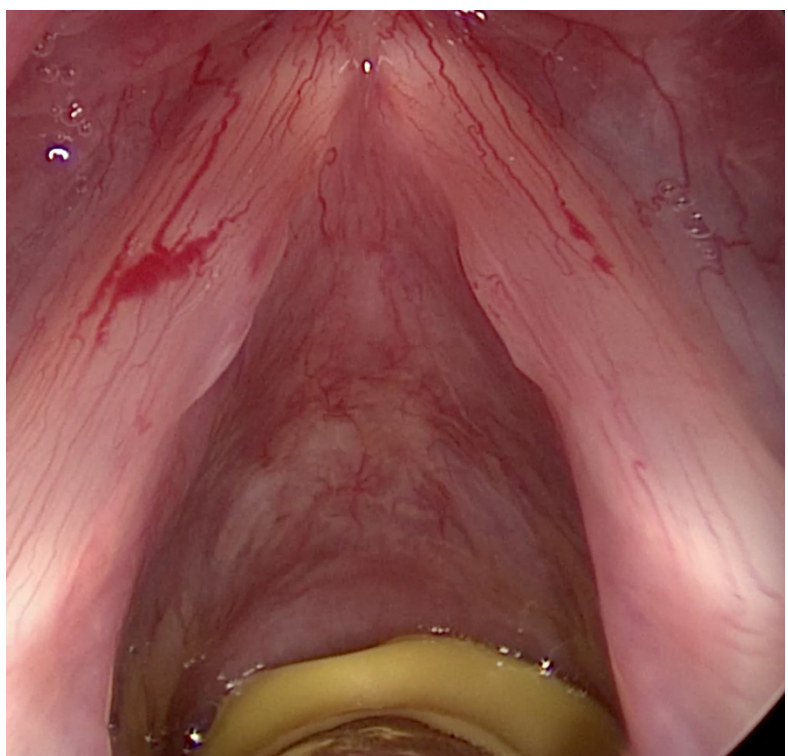

\section{Orgánica}

- Papilomatosis laríngea recurrente juvenil (Figura 2): entidad que debe descartarse frente a un niño con disfonía persistente, dada la posibilidad de progresión a obstrucción de vía aérea. Es provocada por Virus Papiloma Humano(VPH) genotipos 6 y 11. № se conoce del todo la vía de contagio, pero la evidencia orienta a una transmisión materno-fetal por una vía más compleja que solo aquella producida en el canal del parto. El tratamiento es quirúrgico y está orientado a remover estas lesiones verrucosas. El uso de terapia adyuvante con antivirales (Cidofovir) aún es controversial, por la ausencia de ensayos clínicos randomizados. Diversas series de casos han mostrado beneficios con el uso de Cidofovir intralesional $(14,15)$.

\section{Figura $\mathbf{N}^{\mathbf{0}} \mathbf{2}$}

Papilomatosis laringea recurrente. Compromiso bilateral de cuerdas vocales.

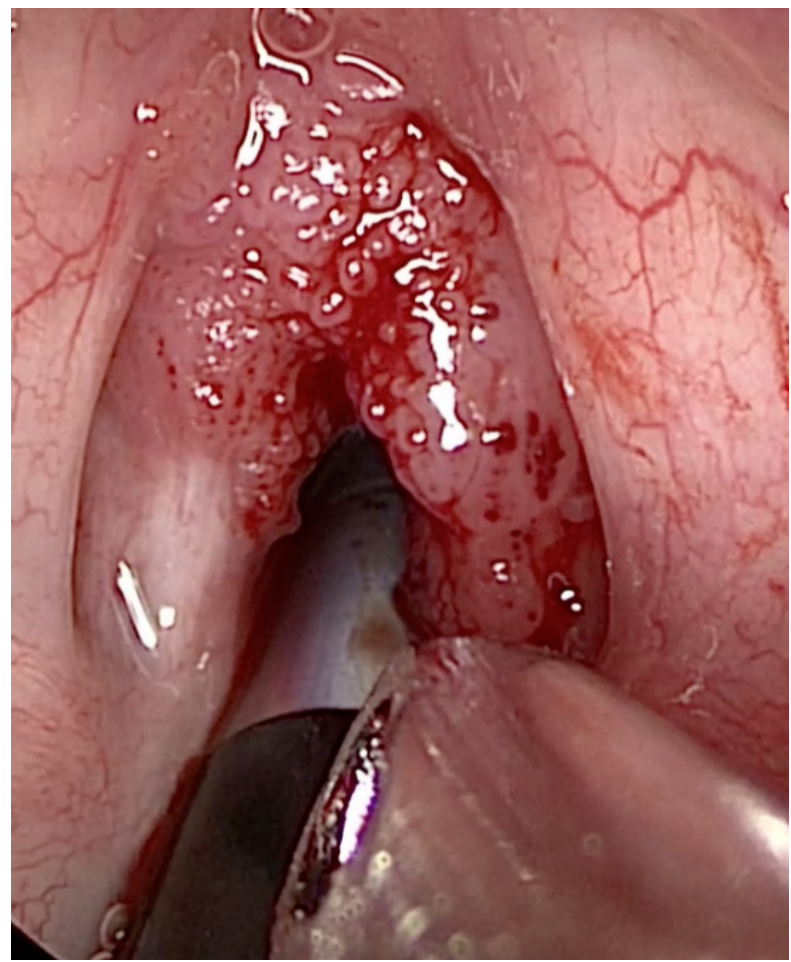

- Parálisis cordales: de etiología variada, desde causa obstétrica (lesión nerviosa por partos distócicos), hasta de origen congénito o secundarias a tumores. Pueden ser uni 0 bilaterales. Las bilaterales con cuerdas en situación paramediana rara vez comprometen la voz o el llanto, pero provocan síntomas respiratorios. El 50\% de estos niños requerirá traqueostomía. Las parálisis unilaterales se manifiestan con disfonía, Ilanto débil y/o aspiración. La mayoría puede tener normalización espontánea de los síntomas con el tiempo, dado por compensación o recuperación de la parálisis. Solo algunos casos requieren técnicas quirúrgicas de medialización de la cuerda parética (16). 
- Alteraciones estructurales mínimas (AEM): descritas por Pontes et al (17), incluyen un grupo de pequeñas imperfecciones de la cubierta de la CV, sin otra alteración morfológica laríngea asociada. Aún se discute si corresponden a una malformación congénita menor 0 si algunas de ellas pudieran ser secundarias a daño de la mucosa laríngea. Se incluyen en este grupo: sulcus vocalis, quistes submucosos, disgenesias vasculares, puentes de mucosa y microwebs (microsinequia anterior). El diagnóstico de estas alteraciones suele ser difícil. Tiende a ser subdiagnósticada y a confundirse con otras patologías secundarias asociadas (pólipos, nódulos vocales, etc). Debe sospecharse ante la presencia de disfonía presente desde edades tempranas, con historia de lesiones benignas recurrentes de las CV o lesiones benignas refractarias a tratamiento. En niños, su diagnóstico es aún más dificultoso y muchas veces este se termina realizando bajo laringoscopía directa. García Martins y colaboradores (18) realizaron videolaringoscopía a 304 pacientes pediátricos disfónicos, encontrando un 21\% de AEM, siendo la más frecuentes los quistes submucosos (15\%) y sulcus vocalis $(3,8 \%)$. Olavarría y colaboradores (19) describen los hallazgos en 48 pacientes pediátricos y adultos con AEM, siendo el sulcus vocalis el más frecuente (64,5\%) y luego quistes (25\%).

La Tabla 1 muestra los diagnósticos de pacientes pediátricos intervenidos por disfonía crónica, en 3 centros de Santiago, entre los años 2004 y 2013 (observaciones no publicadas).

Tabla N ${ }^{0} 1$

Causas de disfonía en 48 pacientes. *

\begin{tabular}{|c|c|}
\hline DIAGNÓSTICO POSOPERATORIO & FRECUENCIA \\
\hline Paresia de cuerda vocal & 2 \\
\hline Quiste submucoso unilateral con lesión contragolpe & 7 \\
\hline Quiste submucoso bilateral & 2 \\
\hline Nódulos cuerda vocal & 4 \\
\hline Nódulos cuerda vocal con microsinequia anterior & 3 \\
\hline Seudoquiste cuarda vocal con lesión contragolpe & 6 \\
\hline Sulcus tipo III & 4 \\
\hline Sulcus tipo II & 8 \\
\hline Puente mucoso & 2 \\
\hline Papiloma laríngeo & 9 \\
\hline
\end{tabular}

* Observaciones no publicadas.

Cabe destacar que la mayoría de estos pacientes habían sido catalogados inicialmente como portadores de NV y reevaluados en unidad de voz por persistencia de síntomas a pesar de terapia fonoaudiológica.

\section{HISTORIA CLÍNICA}

El síntoma de presentación característico en un trastorno de la voz es la "ronquera". Un paso clave en su evaluación es distinguir si el niño presenta un problema de la voz o un problema del habla, la articulación o del lenguaje (3,5-7).
Siempre que sea posible, la información debe ser obtenida de los padres y el niño. La cronología de los síntomas es útil. Si el problema existe desde el nacimiento, es muy probable una patología congénita; sin embargo, la presencia del antecedente de intubación en el período perinatal puede ser sugerente de una patología adquirida, como la estenosis subglótica, la formación de quistes o fibrosis cricoaritenoidea. Cuando el inicio es más tardío, suele estar en relación a una infección respiratoria superior (con laringitis), y que la persistencia de la ronquera sea provocada por el mal uso de voz (3). La presencia de estridor y la disminución de la tolerancia al ejercicio sugieren una patología obstructiva, como la estenosis laríngea o papilomas. Alteraciones en la deglución 0 atoros pueden sugerir la parálisis de alguna CV.

Es necesario preguntar acerca de síntomas de reflujo laringofaríngeo, que pueden causar irritación local y disfonía. La rinitis alérgica con descarga posterior, induce carraspeo, que también puede relacionarse con patología laríngea. La tos persistente puede causar ronquera, la patología respiratoria restrictiva reduce la presión infraglótica afectando la fuerza de la voz y los inhaladores corticoidales utilizados para tratar el asma también puede causar disfonía por atrofia del epitelio vocal (7).

\section{EVALUACIÓN DEL PACIENTE DISFÓNICO}

Todo paciente con disfonía persistente de más de dos semanas debiera ser evaluado por el especialista. Esto, considerando que la causa más frecuente de disfonía aguda en niños y adultos corresponde a laringitis aguda viral, que por lo general se resuelve en un periodo inferior a 14 días. En la evaluación de la disfonía debe realizarse un examen otorrinolaringológico completo, que incluya el examen de oídos y audición. Este debe ser complementado con la evaluación del niño y de sus padres respecto de la percepción de la voz. Una escala útil es la "pediatric Voice Handicap Index (pVHI)" (20) y una escala de "disconfort del tracto vocal" modificada por Mathieson (21).

Existe un subgrupo de pacientes disfónicos que requiere un estudio más profundo. Estos son: Ios que presentan disfonía severa o rebelde a terapia fonoaudiológica, ante la sospecha de patología ominosa, y en casos de disfonía asociada a estridor.

\section{PROCEDIMIENTOS DIAGNÓSTICOS}

\section{Fibro-laringoscopía flexible}

Este examen es capaz de excluir lesiones laríngeas focales, proporcionar imágenes de calidad adecuada sin riesgos asociados relevantes. Se realiza por vía transnasal, con fibra óptica de 2,2 $04.0 \mathrm{~mm}$. Se pudieran realizar en niños de cualquier edad (los más pequeños pudieran ser menos cooperadores). 


\section{Tele-laringoscopía rígida}

Requiere altos niveles de cooperación por parte del menor, siendo utilizado generalmente en los mayores de 6 años. Las imágenes obtenidas son de excelente calidad, dando una información incomparable acerca de la estructura y el movimiento de las CV. Otorga menos información funcional que la fibroscopía.

\section{Estroboscopía}

Consiste en la aplicación de luz estroboscópica durante la evaluación endoscópica del paciente. Permite visualizar con un efecto de "cámara lenta" la vibración de la mucosa de la CV durante la fonación. Es muy útil para identificar pequeñas lesiones en la cubierta del pliegue vocal (22).

\section{Electromiografía laríngea}

Se realiza usando electrodos por vía percutánea cervical en los músculos tiroaritenoideo y cricoaritenoideo posterior. Tiene un rol en niños mayores y adolescentes para el estudio de la función neuromuscular de las CV, por ejemplo, para predecir la recuperación en la parálisis de las CV $(23,24)$.

\section{Laringoscopía directa}

Se realiza en pabellón bajo sedación 0 anestesia general. Mediante un laringoscopio tubular, se canulan los tejidos blandos de la faringe hasta exponer las CV. Puede apoyarse el procedimiento con microscopio. Este examen permite una excelente visualización de las anomalías estructurales, pero una limitada información de su función. Esta evaluación actualmente ha sido desplazada por exámenes menos invasivos, pero tiene gran utilidad aún, principalmente en niños pequeños o aquellos que son poco cooperadores con los otros exámenes. Su principal ventaja, es que permite realizar al mismo tiempo procedimientos terapéuticos.

\section{Análisis acústico de la voz}

En mayores de 4 años es posible realizar el análisis de los parámetros acústicos de la onda vocal. Podemos evaluar la frecuencia fundamental, intensidad, niveles de perturbación de la amplitud o la frecuencia, entre otros. Esto permite contar con datos más objetivos de evaluación que la sola percepción acústica del examinador. Sin embargo, los parámetros de normalidad de estas mediciones aún están en evaluación (25).

Existen otras pruebas que pueden ser consideradas para evaluar la evidencia de reflujo, si bien, no son siempre concluyentes. Estos incluyen el monitoreo de pH (pHmetría) e impedancia intraluminal multicanal para evaluar el reflujo no ácido. La prueba terapéutica anti-reflujo en los casos sospechosos tiene cierta utilidad para algunos especialistas, en vez de realizar procedimientos más invasivos.

\section{ENFOQUE TERAPÉUTICO DEL PACIENTE DISFÓNICO}

Si bien en un grupo minoritario de pacientes pediátricos el tratamiento será primordialmente quirúrgico (papilomas laríngeos, quistes submucosos, entre otros), en la mayoría el tratamiento irá enfocado a manejar los fenómenos de hiperfunción laríngea y mal uso vocal (9). Esta terapia la realizará el fonoaudiólogo. Lamentablemente existe una serie de inconvenientes en este punto: niños poco motivados a la terapia, padres no comprometidos con el tratamiento, fonoaudiólogo con experiencia en patología de la voz pero sin experiencia en manejo con niños, etc.

Se sugiere realizar este tipo de intervenciones terapéuticas después de los 7 años de edad, aunque debe evaluarse cada caso en particular. La adecuada comunicación entre el fonoaudiólogo y el laringólogo es fundamental para el éxito de la terapia y la eventual reevaluación del caso si amerita.

\section{COMENTARIO FINAL}

La disfonía en el niño suele ser un síntoma subestimado. La razón principal es que se asume se trata de una condición benigna asociada al mal uso o abuso vocal. Si bien esto puede ser cierto en la mayoría de los casos, debe realizarse un esfuerzo en establecer un diagnóstico preciso y luego tratar la condición si corresponde. Con el avance de la tecnología endoscópica se puede realizar este tipo de diagnósticos en niños de cualquier edad, con exámenes de gran certeza y poco invasivos, por lo que no existen cuestionamientos razonables para no derivar a este grupo de pacientes al especialista $(1,9)$.

En nuestra casuística pudimos comprobar que, en al menos 11 pacientes, el diagnóstico benigno de nódulos vocales fue reemplazado por diagnósticos tan relevantes y de tratamiento tan específico como parálisis cordal o papiloma laríngeo. Esto se podría explicar por la falta de rigurosidad en evaluación endoscópica 0 por presentarse dificultad técnica para la realización del examen. Las lesiones más frecuentemente encontradas fueron alteraciones estructurales mínimas de la cubierta de la CV (19). Este grupo de pacientes es particularmente interesante dado que se trata, por lo general, de disfonías severas que aparecen muy precozmente en la vida. № existe literatura suficiente ni protocolos terapéuticos específicos hasta la fecha para el enfrentamiento de estas alteraciones. Por otro lado es aún controversial si la resolución temprana de las lesiones encontradas en esta evaluación exhaustiva pueda determinar un pronóstico vocal más favorable.

Los autores declaran no tener conflictos de interés, en relación a este artículo. 


\section{REFERENCIAS}

1. Schwartz SR, Cohen SM, Dailey SH, Rosenfeld RM, Deutsch ES, Gillespie MB et al. Clinical practice guideline: hoarseness (dysphonia). Otolaryngol Head Neck Surg 2009; 141:1-31

2. Tavares EL, Brasolotto A, Santana MF, Padovan CA, Martins RH. Epidemiological study of disphonia in 4-12 years old children. Braz J Otorhinolaryngol 2011;77:736-746

3. Carding PN, Roulstone S, Northstone K; ALSPAC Study Team. The prevalence of childhood dysphonia: a cross sectional study. J Voice 2006;20:623-630

4. Fuchs M, Meuret S, Sthurman N, Schade G. Dysphonia in children and adolescents. HNO 2009; 57:603-614

5. Possamai V, Hartley B. Voice disorders in Children. Pediatr Clin N Am 2013;60: 879-892

6. Dalal PG, Murray D, Messner AH, Feng A, McAllister J, Molter D. Pediatric laryngeal dimensions: an age based analysis. Anesth Analg 2009; 108:1475-1479

7. Hamdan AL, Deeb R, Sibai A, Rameh C, Rifai H, Fayyad J. Vocal characteristics in children with attention deficit hyperactivity disorder. J Voice 2009; 23:190-194

8. Martins RH, Defaveri J, Custódio Domingues MA, de Albuquerque E Silva R, Fabro A. Vocal folds nodules: morphological and inmunohistochemical investigations. J Voice $2010 ; 24: 531-539$

9. Connelly A, Clemente W, Kubba $\mathrm{H}$. Management of dysphonia in children. J Laryngol Otol 2009. 123: 642-647

10. Karkos P, McCormick M. The etiology of vocal folds nodules in adults. Curr Opin Otolaryngol Head Neck Surg 2009;17: 420-423

11. Shah R, Woodnorth G, Glynn A, Glynn A Nuss RC. Pediatric vocal nodules. Int J Pediatr Otorhinolaryngol 2005;60: 903-909

12. Pedersen M, McGlashan J. Surgical vs non surgical interventions for vocal folds nodules. Cochrane Datbase Syst Rev 2012; (6):CD001934

13. Bouchayer M, Cornut G. Microsurgical treatment for benign vocal fold lesions. Folia Phoniatr (Basel)1992; 44:155-184

14. Wierzbicka M, Jackowska J, Bartochowska A, Józefiak A, Szyfter W, Kedzia W. Effectiveness of Cidofovir intralesional treatment in recurrent respiratory papillomatosis. Eur Arch Otorhinolaryngol 2011; 268:130511

15. Pontes P, Weckx LL, Pignatari SS, Fujita RR, Avelino MA, Sato J. Local application of Cidofovir as adjuvant theraphy in recurrent laryngeal papillomatosis in children. Rev Assoc Med Bras 2009; 55: 581-6

16. Daya H, Hosni A, Bejar-Solar I, Evans JN, Bailey CM . Pediatric vocal fold paralysis: a long term retrospective study. Arch Otolaryngol Head Neck Surg 2000; 126: 21-25

17. Pontes P, Behlau M, Goncalves I. Minor structural alterations of the larynx: basic considerations. Acta Awho 1994. 3:2-6

18. García R, Hidalgo C, Zeponi B, Branco A, Mendes E. Dysphonia in children. J Voice 2012; 26: 671-674

19. Olavarría C, Brunetto B, Nazar R, Miranda M, Painepán JC. Alteraciones estructurales mínimas: su rol etiopatogénico en los trastornos vocales. Rev Otorrinolaringol Cir Cabeza Cuello 2004; 64: 7-12

20. Zur KB, Cotton S, Kelchner L, Baker S, Weinrich B, Lee L. Pediatric Voice Handicap Index (pVHI): a new tool for evaluating pediatric dysphonia. Int J Pediatr Otorhinolaryngol. 2007; 71:77-82

21. Mathieson L, Hirani SP, Epstein R, Baken RJ, Wood G, Rubin JS. Laryngeal manual therapy: a preliminary study to examine its treatment effects in the management of muscle tension dysphonia. J Voice 2009;23: 353-366

22. Mackiewics-Nartowics $H$, Sinkiewics A, Bielecka A. Laryngovideostroboscopy in children - diagnostic possibilities and constraints. Int J Pediatr Otorhinolaryngol 2011;75: 1015-1017

23. Maturo SC, Braun N, Brown DJ, Chong PS, Kerschner JE, Hartnick CJ. Intraoperative laryngeal electromyography in children with vocal fold inmovility: results of a multicenter longitudinal study. Arch Otolaryngol Head Neck Surg 2011; 137: 1251-1257

24. AlQudehy Z, Norton J, El-Hakim H. Electromyography in children's laryngeal mobility disorders: a proposed grading system. Arch Otolaryngol Head Neck Surg 2012; 138: 936-941

25. Campisi P, Tewfik TL, Manoukian JJ, Schloss MD, Pelland-Blais E, Sadeghi N. Computer-assisted voice analysis: establishing a pediatric database. Arch Otolaryngol Head Neck Surg 2002; 128: 156-60 